\title{
Cost-Effectiveness of Niraparib and Olaparib as Maintenance Therapy for Patients with Platinum-Sensitive Recurrent Ovarian Cancer
}

\author{
Lixian Zhong, PhD; Anh Thu Tran, BS; Taylor Tomasino, BS; \\ Elizabeth Nugent, MD; and Judith A. Smith, PharmD, BCOP, CPHQ
}

\begin{abstract}
BACKGROUND: The recent approval of olaparib and niraparib as maintenance therapy can significantly affect the management of ovarian cancer. Clinical benefits, however, come with trade-offs in adverse events and costs.

OBJECTIVE: To evaluate the cost-effectiveness of new ovarian cancer polyADP ribose polymerase (PARP) inhibitor therapies, olaparib and niraparib, as maintenance therapy for patients with platinum-sensitive recurrent ovarian cancer.

METHODS: A decision tree model was constructed to evaluate the costs and effectiveness of olaparib and niraparib compared with placebo from a U.S. health care sector perspective. Costs included drug costs and costs of disease monitoring and management of adverse events throughout the treatment course. Costs were estimated from RED B00K, Medicare reimbursement rates, and the literature and reported in 2017 U.S. dollars. Clinical effectiveness was measured in progression-free survival (PFS) life-years based on clinical trial results (NCT00753545, NCT01874353, and NCT01847274). The incremental cost-effectiveness ratio (ICER) was computed by dividing the incremental cost by the incremental effectiveness.

RESULTS: At base case, niraparib was the more effective treatment option with slightly higher PFS, followed by olaparib. The ICERs for niraparib and olaparib compared with common baseline placebo were $\$ 235 \mathrm{~K}$ and \$287K per PFS life-year, respectively, with olaparib extended-dominated by niraparib. Both drugs were associated with lower ICERs in patients with a $g B R C A$ mutation than in patients without a $g B R C A$ mutation. One-way sensitivity analysis suggested that drug prices and PFS could affect ICERs significantly, but the ICERs remained above \$100K per PFS life-year within the plausible ranges of all parameters. Probabilistic sensitivity analysis suggested that niraparib was associated with higher net benefits compared with placebo only when willingness-to-pay (WTP) values were above \$210K per PFS life-year thresholds.
\end{abstract}

CONCLUSIONS: PARP inhibitors niraparib and olaparib will extend PFS in platinum-sensitive recurrent ovarian cancer patients but are also associated with high drug acquisition costs. The base case ICERs were around or above $\$ 250 \mathrm{~K}$ per PFS life-year in this model. No formal cost-effectiveness WTP threshold for health technology assessment exists in the United States. At a reference WTP of $\$ 100 \mathrm{~K}$ per PFS life-year, the PARP inhibitors may not be cost-effective options.

J Manag Care Spec Pharm. 2018;24(12):1219-28

Copyright $\odot 2018$, Academy of Managed Care Pharmacy. All rights reserved.

\section{What is already known about this subject}

The FDA recently approved niraparib and olaparib as maintenance therapies for adult patients with platinum-sensitive recurrent ovarian cancer.

The gains in progression-free survival (PFS) associated with both therapies come with trade-offs in high costs and adverse events, which are important considerations to the health care sector when evaluating the value of these new, innovative therapies.

\section{What this study adds}

In the base case model, when compared with common baseline placebo, the incremental cost-effectiveness ratios (ICER)s for niraparib and olaparib were $\$ 235 \mathrm{~K}$ and $\$ 287 \mathrm{~K}$, respectively, for an additional gain of PFS life-year, with olaparib being extendeddominated by the combination of placebo and niraparib.

In patients with a germline BRCA mutation, the ICERs for niraparib and olaparib compared with placebo were $\$ 197 \mathrm{~K}$ and $\$ 226 \mathrm{~K}$ per PFS life-year, respectively, with ICERs for niraparib and olaparib at $\$ 253 \mathrm{~K}$ and $\$ 328 \mathrm{~K}$ per PFS life-year, respectively, in patients without a gBRCA mutation.

At a willingness-to-pay threshold of $\$ 100 \mathrm{~K}$ per PFS life-year, niraparib and olaparib were less likely to be associated with more net benefit than placebo.

( varian cancer is one of the leading causes of death in women worldwide and the most fatal gynecologic cancer in the United States, with a mortality rate of 7.4 per 100,000., In the United States alone, it accounted for an estimated 22,240 new cases and 14,070 deaths in 2018. ${ }^{3}$

About $80 \%$ of ovarian cancer patients are at advanced disease stage 3 or 4 at diagnosis, when tumors have spread to the regional lymph nodes or metastasized to the organs outside of the abdomen area, respectively., ${ }^{2,4}$ The initial treatment for these patients may consist of surgical staging, cytoreduction, and first-line platinum-based chemotherapy. Most of these patients initially achieve some clinical response to the chemotherapy but will eventually relapse. ${ }^{5}$

The risk of relapse after the initial therapy can be as high as $80 \%-85 \%$ in patients at stage 3 or $4 .{ }^{6}$ The management of recurrent ovarian cancer is based on many factors, including the duration of the platinum-free interval, adverse events, 


\section{Cost-Effectiveness of Niraparib and Olaparib as Maintenance Therapy}

for Patients with Platinum-Sensitive Recurrent Ovarian Cancer

performance status, histology, disease burden, and tumor biomarkers, such as BRCA mutation status. ${ }^{\top}$

For patients with recurrent platinum-sensitive ovarian cancer, maintenance treatment with targeted agents such as the angiogenesis inhibitor bevacizumab has resulted in improved progression-free survival (PFS) ${ }^{8,9}$ More recently, 2 poly-ADP ribose polymerase (PARP) inhibitors, niraparib (Zejula, Tesaro) and olaparib (Lynparza, AstraZeneca), have also been approved by the U.S. Food and Drug Administration (FDA) as maintenance therapy for this patient population. In a randomized, double-blind, phase 2 trial (Study 19, NCT00753545), olaparib maintenance therapy resulted in 8.4 months of median PFS versus 4.8 months in placebo (hazard ratio $[\mathrm{HR}]=0.35,95 \%$ confidence interval $[\mathrm{CI}]=0.25-0.49) .{ }^{10}$ In an international, multicenter, double-blind, randomized, placebo-controlled phase 3 trial (SOLO-2 trial, NCT01874353), the median PFS in a cohort with BRCA1 or BRCA2 germline mutations ( $g B R C A)$ was significantly longer in the olaparib arm at 19.1 months compared with 5.5 months in the placebo arm $(\mathrm{HR}=0.30,95 \%$ $\mathrm{CI}=0.22-0.41) .{ }^{11}$ Also, in a randomized, double-blind, phase 3 trial (NOVO trial, NCT01847274), niraparib maintenance therapy resulted in 21.0 months of PFS versus 5.5 months of PFS in placebo in a $g B R C A$ cohort $(\mathrm{HR}=0.27,95 \% \mathrm{CI}=0.17$ 0.41 ) and 9.3 months versus 3.9 months in the non-gBRCA cohort $(\mathrm{HR}=0.45,95 \% \mathrm{CI}=0.34-0.61){ }^{12}$

While the PARP inhibitor drugs have significantly extended PFS in the clinical trial setting, they are also associated with high costs as translated into use in clinical practice. The 2017 wholesale acquisition costs (WAC prices) for olaparib and niraparib were $\$ 13,482$ and $\$ 14,750$, respectively, for a 30 -day supply. ${ }^{13}$ In addition, there are costs associated with routine therapy monitoring and management of adverse events associated with treatment. The purpose of this study was to evaluate the cost-effectiveness of newly approved olaparib and niraparib as maintenance therapies in platinum-sensitive recurrent ovarian cancer patients.

\section{Methods}

\section{Model}

A decision analysis model (Figure 1) was constructed to estimate the cost-effectiveness of olaparib and niraparib compared with placebo in treating platinum-sensitive recurrent ovarian cancer patients. The decision tree was composed of decision nodes, which laid out alternatives to be compared, and chance nodes, which laid out possible outcomes due to uncertainty. The decision nodes included olaparib, niraparib, and placebo (observation) as treatment options. The first chance node of each treatment option was stratified by gBRCA status. The clinical trials reported differential PFS with regard to gBRCA status, and patients with $g B R C A$ mutations tended to benefit more from the treatments. The second chance node indicated the probabilities of dose reduction due to adverse events, allowing the model to reflect differences in total drug costs, as well as the costs for mitigating adverse events.

The analysis was conducted from a U.S. health care sector perspective. Costs were reported in 2017 U.S. dollars, and health outcomes were assessed using PFS life-years. Model inputs for clinical outcomes were based on published results of clinical trials: olaparib Study 19 (NCT00753545), olaparib SOLO-2 trial (NCT01874353), and niraparib NOVO trial (NCT01847274). ${ }^{10-12}$ Costs were estimated using standard sources (see Cost Estimates section). All modeling and computation were conducted using TreeAge Pro Software, 2014 (TreeAge Software, Williamston, MA).

\section{Patient Population}

The analysis presented in this study applies to adult patients with recurrent epithelial ovarian, fallopian tube, or primary peritoneal cancer, who have received previous treatments of platinum-based chemotherapy and were in a complete or partial response to the most recent chemotherapy.

\section{Time Horizon and Discounting}

The costs and outcomes were measured until disease progression or death. Because of the relatively short time horizon, discounting was not applied in computing costs and outcomes.

\section{Cost Estimates}

The analysis included drug costs, costs of disease monitoring, and costs of management of adverse events (AEs) throughout the treatment course. Drug costs were obtained from the 2017 RED BOOK using WAC prices. ${ }^{14}$ Doses were adjusted to reflect real-world use due to treatment side effects. Clinical laboratory test costs were obtained from the 2017 Medicare Clinical Laboratory Fee Schedule. Imaging and other health care services and procedure costs were obtained from the 2017 Medicare Physician Fee Schedule. Costs of mitigating severe AEs were obtained from the literature and converted to 2017 U.S. dollars using the medical component of the Consumer Price Index. ${ }^{15}$ All costs were presented in 2017 U.S. dollars (Table 1).

AEs during treatment also increase costs for payers. This study included costs connected with grade 3 and greater AEs associated with the active treatments. ${ }^{16}$ Grade 3 and greater AEs require significant intervention and may require hospitalization or prolongation of hospitalization. Grade 1 or 2 AEs are generally considered to be mild or moderate with minimal intervention required; thus, costs associated with grade 1 or 2 AEs were not included. The AEs included in this analysis were modeled from the clinical trials (Table 1).

\section{Effectiveness Estimates}

PFS was the primary endpoint of the clinical trials (NCT00753545, NCT01874353, and NCT01847274). ${ }^{10-12}$ In this 


\section{Cost-Effectiveness of Niraparib and Olaparib as Maintenance Therapy}

for Patients with Platinum-Sensitive Recurrent Ovarian Cancer

\section{FIGURE 1 The Decision Tree Model Structure}

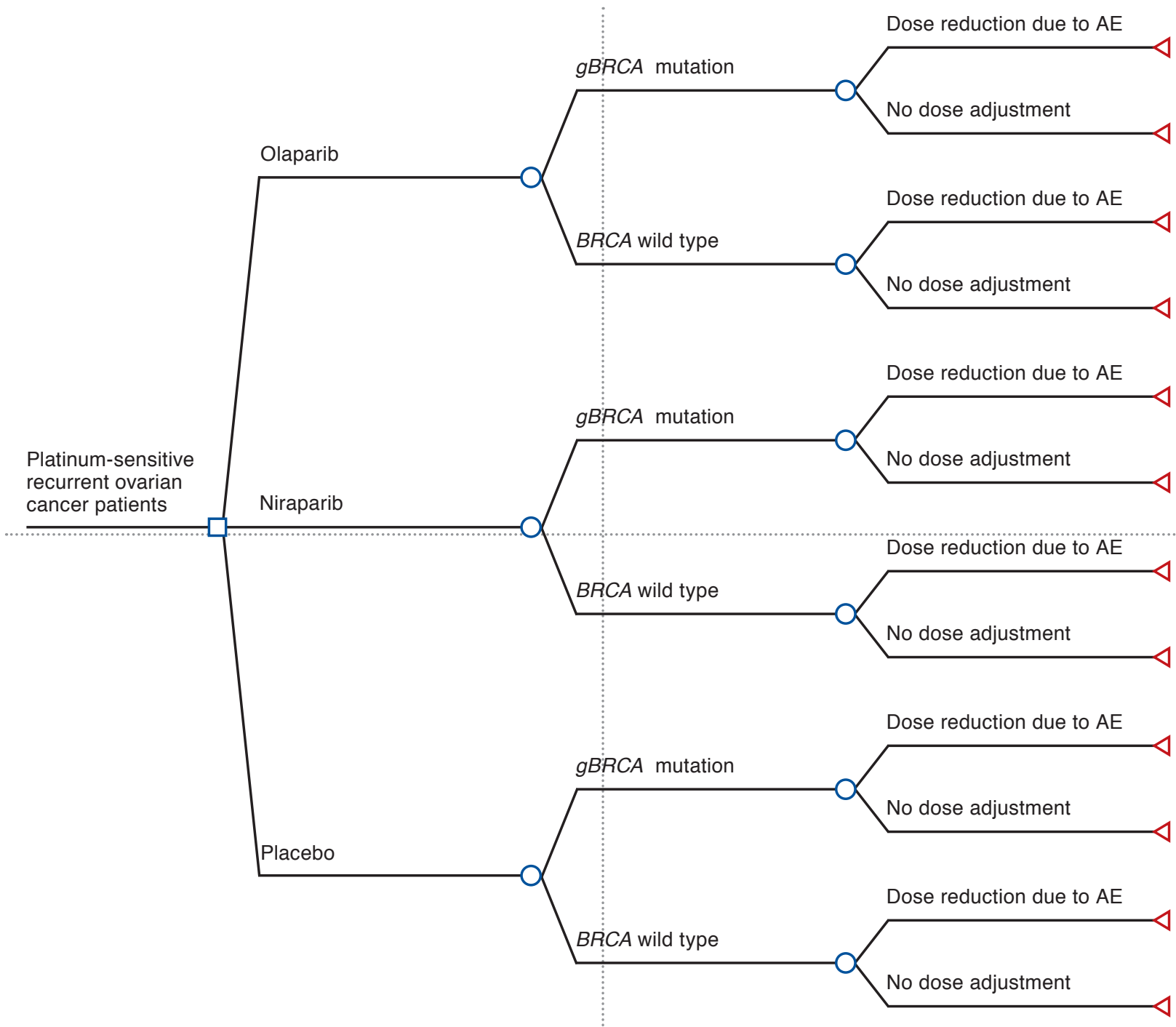

Note: Choice nodes included olaparib, niraparib, and placebo. Decision nodes included patient BRCA mutation status and dose adjustment status. Probabilities, costs, and outcomes were assigned to each branch based on model inputs in Table 1.

$A E=$ adverse event; $g B R C A=$ germline $B R C A$ mutation.

study, PFS life-years were used as the effectiveness measure for the base case analysis. The sensitivity analysis also computed quality-adjusted PFS (QA-PFS) life-years as an outcome measure. QA-PFS was measured by multiplying the PFS life-years with health-state utility values (HSUV): QA-PFS = PFS $\times$ HSUV. PFS life-years were directly obtained from reported median PFS in clinical trials (Table 1). Health-related quality of life was incorporated into the analysis using HSUV reported in the literature. By applying HSUV, PFS was downwardly adjusted by HSUV to reflect the effect of quality of life in these patients.

\section{Cost-Effectiveness Analysis}

Costs were defined as the total cost per strategy and included pharmacy costs and medical costs. The effectiveness was defined as PFS in the base case analysis and included QA-PFS as part of the sensitivity analysis. All 3 treatment strategies were ranked based on costs from low to high. Incremental costs and effectiveness were computed against the next costly option. Incremental cost-effectiveness ratio (ICER) was computed as the incremental cost per incremental PFS:

$$
\text { ICER }=\left(\text { Cost }_{1}-\text { Cost }_{2}\right) \div\left(\text { Effectiveness }_{1}-\text { Effectiveness }_{2}\right) \text {. }
$$




\section{Cost-Effectiveness of Niraparib and Olaparib as Maintenance Therapy for Patients with Platinum-Sensitive Recurrent Ovarian Cancer}

TABLE 1 Decision Tree Model Input

\begin{tabular}{|c|c|c|c|}
\hline Model Inputs & $\begin{array}{c}\text { Base-Case } \\
\text { Value }\end{array}$ & Distribution & Notes \\
\hline \multicolumn{4}{|l|}{ Costs, \$ } \\
\hline \multicolumn{4}{|l|}{ Drug cost per 30-day supply } \\
\hline Niraparib & 14,750 & gamma & 2017 RED BOOK WAC price. Pkg size: 90s of 100 mg13 \\
\hline Olaparib & 13,482 & gamma & 2017 RED BOOK WAC price. Pkg size: 120s of $150 \mathrm{mg} \mathrm{tab}{ }^{13}$ \\
\hline \multicolumn{4}{|l|}{ Monitoring costs } \\
\hline Level 4 office visit ${ }^{\mathrm{a}}$ & 108.74 & gamma & Once per cycle. ${ }^{b}$ Medicare Physician Fee Schedule (HCPCS 99214) ${ }^{32}$ \\
\hline CA 125 test $^{\mathrm{c}}$ & 28.55 & gamma & Once per cycle. Medicare Physician Fee Schedule (HCPCS 86304)32 \\
\hline Complete blood count with differential & 10.66 & gamma & Once per cycle. Medicare Physician Fee Schedule (HCPCS 85025)32 \\
\hline Comprehensive metabolic panel & 14.49 & gamma & Once per cycle. Medicare Physician Fee Schedule (HCPCS 80053)32 \\
\hline Abdomen and pelvis CT with contrast & 315.46 & gamma & Once every 3 cycles. Medicare Physician Fee Schedule (HCPCS 74177) 32 \\
\hline \multicolumn{4}{|c|}{ Cost of managing grade $3 / 4$ adverse events per episoded } \\
\hline Anemia & 7,680 & gamma & Reference 33 \\
\hline Neutropenia & 13,248 & gamma & Reference 33 \\
\hline Thrombocytopenia & 5,737 & gamma & Reference 33 \\
\hline Small intestinal obstruction & 29,831 & gamma & Reference 34 \\
\hline \multicolumn{4}{|l|}{ Probabilities, \% } \\
\hline Rate of $g B R C A$ mutation & 15.00 & beta & References 35-37 \\
\hline \multicolumn{4}{|c|}{ Probabilities of severe grade $3 / 4$ adverse events } \\
\hline \multicolumn{4}{|c|}{ Niraparib } \\
\hline Anemia & 25.30 & beta & Reference 12 \\
\hline Neutropenia & 19.60 & beta & Reference 12 \\
\hline Thrombocytopenia & 33.80 & beta & Reference 12 \\
\hline \multicolumn{4}{|l|}{ Olaparib } \\
\hline Anemia & 19.00 & beta & Reference 11 \\
\hline Neutropenia & 5.00 & beta & Reference 11 \\
\hline \multicolumn{4}{|l|}{ Probabilities of dose adjustment } \\
\hline Niraparib & 66.50 & beta & Reference 12; reduced from $300 \mathrm{mg}$ to $200 \mathrm{mg}$ daily \\
\hline Olaparib & 25.00 & beta & Reference 11; reduced from $300 \mathrm{mg}$ to $250 \mathrm{mg}$ twice daily \\
\hline \multicolumn{4}{|l|}{ Progression-free survival (months) } \\
\hline \multicolumn{4}{|l|}{ Niraparib } \\
\hline gBRCA & 21.00 & normal & Reference 12 \\
\hline Non-gBRCA & 9.30 & normal & Reference 12 \\
\hline \multicolumn{4}{|l|}{ Olaparib } \\
\hline gBRCA & 19.10 & normal & Reference 11 \\
\hline Non-gBRCA & 7.40 & normal & Reference 38 \\
\hline \multicolumn{4}{|l|}{ Placebo } \\
\hline$g B R C A$ & 5.50 & normal & Reference 11,12 \\
\hline Non-gBRCA & 3.80 & normal & Reference 12 \\
\hline \multicolumn{4}{|l|}{ Utility values } \\
\hline gBRCA subpopulation & 0.768 & beta & Reference 30 \\
\hline Non-gBRCA subpopulation & 0.800 & beta & Reference 30 \\
\hline
\end{tabular}

aThe second highest level of care for established office patients.

${ }^{b}$ Defined as a 4-week treatment period consistent with the clinical trials.

Cancer Antigen-125 test.

dAdjusted to 2017 U.S. dollars using the medical component of the Consumer Price Index.

$C T=$ computed tomography; gBRCA = germline BRCA mutation; HCPCS=Healthcare Common Procedure Coding System; WAC= wholesale acquisition cost

\section{Sensitivity Analysis}

One-way and probabilistic sensitivity analyses were conducted to validate the model's robustness. One-way sensitivity analysis was conducted by varying the value of 1 variable at a time within its plausible range, which was set to be $\pm 25 \%$ of the base case value. Probabilistic sensitivity analysis was conducted by varying all variables at the same time by running 1,000 Monte Carlo simulations. Beta distributions were assigned to probabilities; HSUVs gamma distributions were assigned to costs; and normal distributions were assigned to PFS estimates. No 


\section{Cost-Effectiveness of Niraparib and Olaparib as Maintenance Therapy for Patients with Platinum-Sensitive Recurrent Ovarian Cancer}

\section{TABLE 2 Cost-Effectiveness Analysis Results}

\begin{tabular}{|c|c|c|c|c|c|c|}
\hline Treatment Option & $\begin{array}{c}\text { Cost } \\
(\$, \text { in 000s) }\end{array}$ & $\begin{array}{c}\text { Total Effect } \\
\text { (PFS Life-Year) }\end{array}$ & $\begin{array}{c}\text { incrCost } \\
(\$, \text { in } 000 s)\end{array}$ & $\begin{array}{c}\text { incrEff } \\
\text { (PFS Life-Year) }\end{array}$ & $\begin{array}{l}\text { ICER Compared } \\
\text { with Next } \\
\text { Costly Option } \\
\text { (\$1,000/PFS } \\
\text { Life-Year) }\end{array}$ & $\begin{array}{c}\text { ICER } \\
\text { Compared with } \\
\text { Placebo } \\
(\$ 1,000 / \text { PFS } \\
\text { Life-Year })\end{array}$ \\
\hline \multicolumn{7}{|c|}{ A. Base-case cost-effectiveness in platinum-sensitive recurrent ovarian cancer patients } \\
\hline Placebo & 1.2 & 0.34 & - & - & - & - \\
\hline Olaparib & 123.2 & 0.76 & 122.0 & 0.43 & 287 & 287 \\
\hline Niraparib & 138.0 & 0.92 & 14.8 & 0.16 & 93 & 235 \\
\hline \multicolumn{7}{|c|}{ B. Base-case cost-effectiveness in patients with gBRCA mutations } \\
\hline Placebo & 1.6 & 0.46 & - & - & - & - \\
\hline Niraparib & 256.3 & 1.75 & 254.7 & 1.29 & 197.2 & 197 \\
\hline Olaparib (dominated) & 257.1 & 1.59 & 0.8 & -0.16 & -5.3 & 226 \\
\hline \multicolumn{7}{|c|}{ C. Base-case cost-effectiveness in patients without gBRCA mutations } \\
\hline Placebo & 1.1 & 0.32 & - & - & - & - \\
\hline Olaparib & 99.6 & 0.62 & 98.5 & 0.30 & 328 & 328 \\
\hline Niraparib & 117.1 & 0.78 & 17.5 & 0.46 & 111 & 253 \\
\hline Treatment Option & $\begin{array}{c}\text { Cost } \\
(\$, \text { in } 000 s)\end{array}$ & $\begin{array}{c}\text { Total Effect } \\
\text { (QA-PFS } \\
\text { Life-Year) }\end{array}$ & $\begin{array}{c}\text { incrCost } \\
(\$, \text { in } 000 s)\end{array}$ & $\begin{array}{c}\text { incrEff } \\
\text { (QA-PFS } \\
\text { Life-Year) }\end{array}$ & $\begin{array}{c}\text { ICER Compared } \\
\text { with Next } \\
\text { Costly Option } \\
\text { (\$1,000/QA-PFS } \\
\text { Life-Year) }\end{array}$ & $\begin{array}{c}\text { ICER } \\
\text { Compared with } \\
\text { Placebo } \\
\text { (\$1,000/QA-PFS } \\
\text { Life-Year) }\end{array}$ \\
\hline \multicolumn{7}{|c|}{ D. Second model to include utilities in outcome measurement of QA-PFS life-year } \\
\hline Placebo & 1.2 & 0.27 & - & - & - & - \\
\hline Olaparib & 123.2 & 0.60 & 122.0 & 0.33 & 365 & 365 \\
\hline Niraparib & 138.0 & 0.73 & 14.8 & 0.13 & 117 & 297 \\
\hline
\end{tabular}

interactions were assumed. Cost-effectiveness acceptability curves were also computed based on the probabilistic sensitivity analysis. The net monetary benefit (NMB) of each treatment strategy was computed using $\mathrm{NMB}=W \times E-C$, and the net health benefit (NHB) was computed using $\mathrm{NHB}=E-C \div W$, where $C$ is the costs associated with each treatment option, $E$ is the effectiveness measure in PFS life-years, and $W$ is the willingness-to-pay (WTP) amount varied along a continuum. For each simulation iteration, NMB (or NHB) could be calculated at different WTP levels. The treatment associated with the highest NMB (or NHB) was considered the most cost-effective option at given WTP levels. Over 1,000 iterations, the probabilities of each treatment option, as the most cost-effective treatment option, were plotted against WTP as the cost-effectiveness acceptability curves. In addition to the one-way and probabilistic sensitivity analyses, a second model was run with HSUV adjustment of PFS to compute QA-PFS as part of the sensitivity analysis.

\section{Results}

\section{Base-Case Analysis}

The costs for a 30-day supply were $\$ 13,482$ for olaparib and $\$ 14,750$ for niraparib, following the recommended dosing regimen by the manufacturers. In the model, we adjusted drug utilization using dose adjustment data reported in the clinical trials. The costs for disease monitoring and management of AEs were also included in computing the total cost but were relatively insignificant compared with drug costs (Table 1).

In the base case (Table 2A), niraparib $(\$ 138.0 \mathrm{~K})$ was the most costly option, followed by olaparib $(\$ 123.2 \mathrm{~K})$ and placebo $(\$ 1.2 \mathrm{~K})$. Drug costs for olaparib and niraparib contributed to the largest portion of total costs. Niraparib was associated with the longest PFS life-years (0.92), followed by olaparib (0.76) and placebo (0.34).

ICER was used to measure the cost-effectiveness of these new drugs. It measured the additional costs associated with 1 additional unit of effectiveness, which was 1 PFS life-year in this case. In the base-case model, when compared with common baseline placebo, the ICERs were $\$ 287 \mathrm{~K}$ for olaparib and $\$ 235 \mathrm{~K}$ for niraparib for an additional gain of a PFS life-year, respectively. When the treatment options were ranked from low costs to high costs, the ICERs were \$93K per PFS life-year for niraparib compared with olaparib and $\$ 287 \mathrm{~K}$ per PFS lifeyear for olaparib compared with placebo (Table 2A).

It should be noted that olaparib was extended-dominated by combinations of placebo and niraparib, meaning that mathematically at population level, using niraparib in subsets of patients could be associated with more total PFS life-years 


\section{Cost-Effectiveness of Niraparib and Olaparib as Maintenance Therapy for Patients with Platinum-Sensitive Recurrent Ovarian Cancer}

TABLE 3 One-Way Sensitivity Analysis for Key Variables

\begin{tabular}{|c|c|c|c|c|c|c|c|}
\hline Variable & Value & Strategy & $\begin{array}{c}\text { Cost } \\
(\$, \text { in } 000 s) \\
\end{array}$ & $\begin{array}{c}\text { incrCost } \\
(\$, \text { in } 000 s)\end{array}$ & $\begin{array}{c}\text { Total Effect } \\
\text { (PFS Life-Year) } \\
\end{array}$ & $\begin{array}{c}\text { incrEff } \\
\text { (PFS Life-Year) }\end{array}$ & $\begin{array}{c}\text { ICER } \\
\text { (\$1,000/PFS } \\
\text { Life-Year) }\end{array}$ \\
\hline \multirow[t]{6}{*}{ Olaparib, WAC price (\$) } & \multirow{3}{*}{$\begin{array}{c}\$ 10,111.5 \\
(-25 \%)\end{array}$} & Placebo & 1.2 & - & 0.34 & - & - \\
\hline & & Olaparib & 93.3 & 92.2 & 0.76 & 0.42 & 216.8 \\
\hline & & Niraparib & 138.0 & 44.7 & 0.92 & 0.16 & 282.1 \\
\hline & \multirow{3}{*}{$\begin{array}{c}\$ 16,852.5 \\
(+25 \%)\end{array}$} & Placebo & 1.2 & - & 0.34 & - & - \\
\hline & & Niraparib & 138.0 & 136.8 & 0.92 & 0.58 & 234.6 \\
\hline & & Olaparib & 153.1 & 15.1 & 0.76 & -0.16 & dominated \\
\hline \multirow[t]{6}{*}{ Niraparib, WAC price $(\$)$} & \multirow{3}{*}{$\begin{array}{c}\$ 11,062.5 \\
(-25 \%)\end{array}$} & Placebo & 1.2 & - & 0.34 & - & - \\
\hline & & Niraparib & 105.9 & 104.8 & 0.92 & 0.58 & 179.6 \\
\hline & & Olaparib & 123.2 & 17.3 & 0.76 & -0.16 & dominated \\
\hline & \multirow{3}{*}{$\begin{array}{c}\$ 18,437.5 \\
(+25 \%)\end{array}$} & Placebo & 1.2 & - & 0.34 & - & - \\
\hline & & Olaparib & 123.2 & 122.1 & 0.76 & 0.42 & 287.2 \\
\hline & & Niraparib & 170.1 & 46.9 & 0.92 & 0.16 & 295.9 \\
\hline \multirow[t]{6}{*}{ Olaparib, PFS non-gBRCA (year) } & \multirow{3}{*}{$\begin{array}{l}0.4625 \\
(-25 \%)\end{array}$} & Placebo & 1.2 & - & 0.34 & - & - \\
\hline & & Olaparib & 102.2 & 101.1 & 0.63 & 0.29 & 343.8 \\
\hline & & Niraparib & 138.0 & 35.8 & 0.92 & 0.29 & 123.6 \\
\hline & \multirow{3}{*}{$\begin{array}{l}0.77083 \\
(+0.25 \%)\end{array}$} & Placebo & 1.2 & - & 0.34 & - & - \\
\hline & & Niraparib & 138.0 & 136.8 & 0.92 & 0.58 & 234.6 \\
\hline & & Olaparib & 144.2 & 6.2 & 0.89 & -0.03 & dominated \\
\hline \multirow[t]{6}{*}{ Olaparib, PFS gBRCA (year) } & \multirow{3}{*}{$\begin{array}{c}1.19375 \\
(-25 \%)\end{array}$} & Placebo & 1.2 & - & 0.34 & - & - \\
\hline & & Olaparib & 113.7 & 112.5 & 0.70 & 0.36 & 307.9 \\
\hline & & Niraparib & 138.0 & 24.3 & 0.92 & 0.22 & 111.6 \\
\hline & \multirow{3}{*}{$\begin{array}{l}1.98958 \\
(+25 \%)\end{array}$} & Placebo & 1.2 & - & 0.34 & - & - \\
\hline & & Olaparib & 132.8 & 131.6 & 0.82 & 0.48 & 271.6 \\
\hline & & Niraparib & 138.0 & 5.2 & 0.92 & 0.10 & 52.8 \\
\hline \multirow[t]{6}{*}{ Niraparib, PFS non-gBRCA (year) } & \multirow{3}{*}{$\begin{array}{c}0.58125 \\
(-25 \%)\end{array}$} & Placebo & 1.2 & - & 0.34 & - & - \\
\hline & & Niraparib & 114.5 & 113.3 & 0.76 & 0.42 & 270.7 \\
\hline & & Olaparib & 123.2 & 874.0 & 0.76 & 0.01 & $1,375.3$ \\
\hline & \multirow{3}{*}{$\begin{array}{c}0.96875 \\
(+25 \%)\end{array}$} & Placebo & 1.2 & - & 0.34 & - & - \\
\hline & & Olaparib & 123.2 & 122.1 & 0.76 & 0.42 & 287.2 \\
\hline & & Niraparib & 161.5 & 38.3 & 1.09 & 0.33 & 118.5 \\
\hline \multirow[t]{6}{*}{ Niraparib, PFS gBRCA (year) } & \multirow{3}{*}{$\begin{array}{l}1.3125 \\
(-25 \%)\end{array}$} & Placebo & 1.2 & - & 0.34 & - & - \\
\hline & & Olaparib & 123.2 & 122.1 & 0.76 & 0.42 & 287.2 \\
\hline & & Niraparib & 128.6 & 5.4 & 0.86 & 0.10 & 58.3 \\
\hline & \multirow{3}{*}{$\begin{array}{l}2.1875 \\
(+25 \%)\end{array}$} & Placebo & 1.2 & - & 0.34 & - & - \\
\hline & & Olaparib & 123.2 & 122.1 & 0.76 & 0.42 & 287.2 \\
\hline & & Niraparib & 147.4 & 24.1 & 0.99 & 0.23 & 107.8 \\
\hline
\end{tabular}

gBRCA = germline BRCA mutation; ICER = incremental cost-effectiveness ratio; incrCost = incremental cost; incrEff = incremental effectiveness; PFS = progression-free survival; $W A C=$ wholesale acquisition costs.

and yet less costly compared with treating all patients with olaparib.

How gBRCA mutation status might affect ICERs was also examined. Both olaparib and niraparib extended PFS better in patients with a gBRCA mutation. The ICERs for niraparib and olaparib compared with common baseline placebo were \$226K and $\$ 197 \mathrm{~K}$ per PFS life-year, respectively, in patients with a gBRCA mutation (Table 2B). The ICERs were $\$ 328 \mathrm{~K}$ and $\$ 253 \mathrm{~K}$ per PFS life-year, respectively, in patients without a $g B R C A$ mutation (Table 2C). This result suggests that these drugs cost less to gain an additional PFS life-year in patients with a $g B R C A$ mutation. It should be noted that when treating patients with a gBRCA mutation, niraparib dominated olaparib in our model with lower costs and better outcomes.

No formal WTP threshold for health technology assessment exists in the United States, and $\$ 100 \mathrm{~K}$ per quality-adjusted life-year has been used as a reference bar. ${ }^{17}$ If tentatively using $\$ 100 \mathrm{~K}$ per PFS life-year as a reference WTP threshold, neither of these new treatment options would be considered cost-effective, since they both cost more than $\$ 200 \mathrm{~K}$ to gain 1 additional PFS life-year compared with placebo. The ICERs were still above $\$ 100 \mathrm{~K}$ per PFS life-year even in gBRCA patients. 


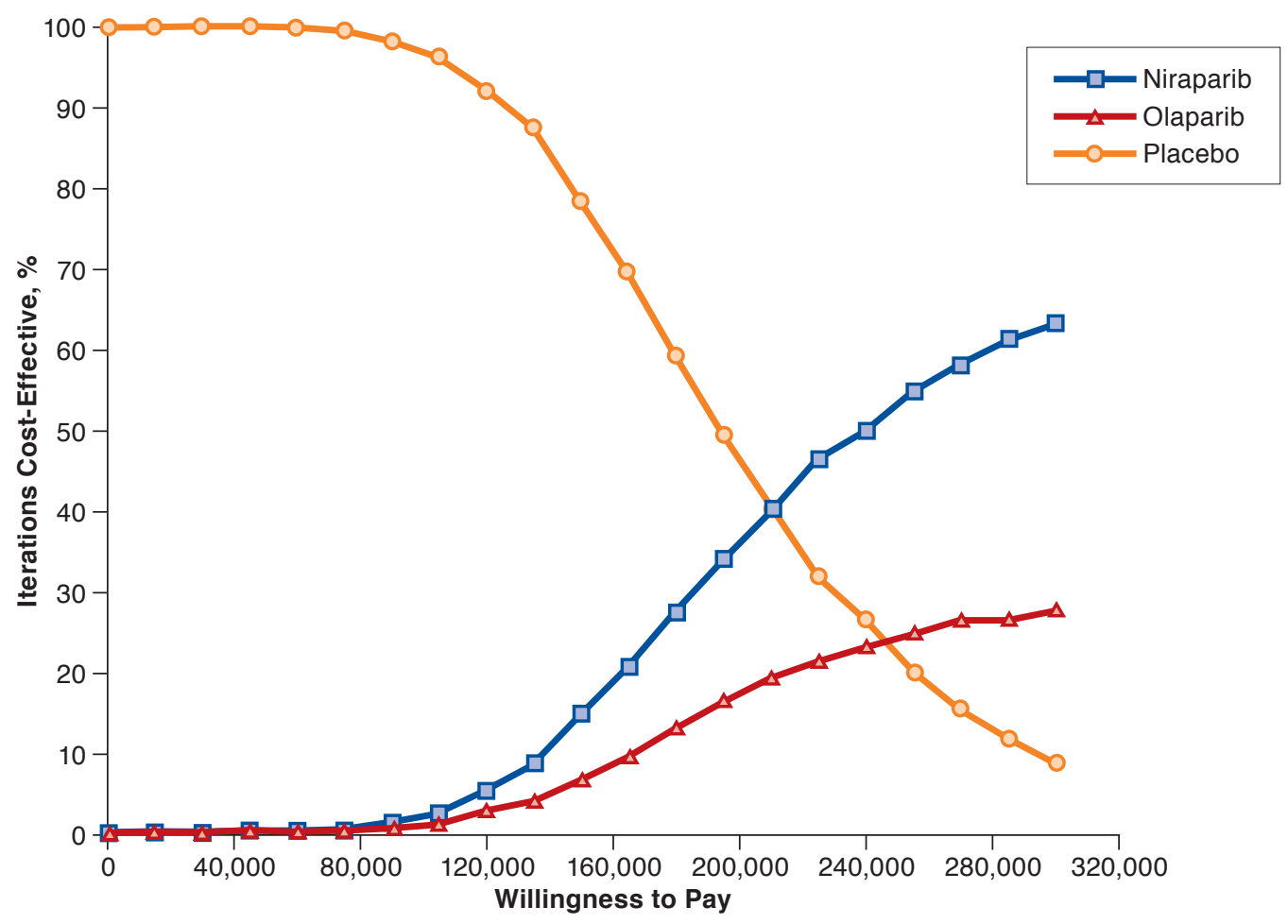

Note: A probabilistic sensitivity analysis with 1,000 Monte Carlo simulations was performed. The curves represent the percentage of times each treatment option had the highest net health benefit at different WTP thresholds. The curve with the higher percentage is considered to be more cost-effective at each WTP value. At a WTP of $\$ 100,000$ per PFS life-year, neither of the PARP inhibitors were cost-effective compared with placebo. When WTP was over $\$ 210,000$ per PFS life-year, niraparib surpassed placebo to become a more cost-effective option.

$P A R P=$ poly $-A D P$ ribose polymerase $; P F S=$ progression-free survival; $W T P=$ willingness to pay.

\section{Sensitivity Analysis}

A Second Cost-Effectiveness Model. In the base-case analysis, the effectiveness measure was PFS life-years. Because of the lack of trial-specific HSUVs, QA-PFS was not computed in the base case. In the sensitivity analysis, a second model was constructed to tentatively incorporate HSUVs from the literature and downwardly adjust PFS to compute QA-PFS as the effectiveness measure. Niraparib was associated with 0.73 QA-PFS life-years, followed by 0.60 QA-PFS life-years for olaparib and 0.27 QA-PFS life-years for placebo. When compared with common baseline placebo, ICERs for olaparib and niraparib were $\$ 365 \mathrm{~K}$ and $\$ 297 \mathrm{~K}$, for an additional gain of a QA-PFS life-year, respectively. The ICER was $\$ 117 \mathrm{~K}$ per QA-PFS life-year for niraparib compared with olaparib. For olaparib, the ICER was \$365K per QA-PFS life-year compared with placebo (Table 2D). As in the base-case model, olaparib was extended-dominated by niraparib.

One-Way Sensitivity Analysis. One-way sensitivity analyses were performed to evaluate the effect of the variation of each individual variable on the ICER. The variables were varied within the plausible range, which was set to be $\pm 25 \%$ of the point estimate of each variable at the base case. In general, the model was robust within the plausible range of variables (Table 3). Changes in drug prices and PFS times could affect ICERs significantly, but they remained above $\$ 100 \mathrm{~K}$ per PFS life-year when compared with placebo.

Probabilistic Sensitivity Analysis. Probabilistic sensitivity analysis was conducted by varying all variables at the same time following each individual variable's distribution. No interactions between variables were assumed. The results of 1,000 Monte Carlo simulations were plotted in the cost-effectiveness plane. The cost-effectiveness acceptability curves (CEACs) of the 3 treatment options (niraparib, olaparib, or placebo) were generated from the probabilistic sensitivity analysis. CEACs present uncertainty as the probability that each alternative has the greatest net benefit, which can be measured in either NMB or NHB, as a function of the WTP. The CEACs showed that niraparib and olaparib were less likely to be associated with 


\section{Cost-Effectiveness of Niraparib and Olaparib as Maintenance Therapy}

for Patients with Platinum-Sensitive Recurrent Ovarian Cancer

more net benefit than placebo below a WTP of $\$ 210 \mathrm{~K}$. Only above $\$ 210 \mathrm{~K}$ did niraparib have an advantage over placebo as the treatment option with the highest net benefit (Figure 2).

\section{Discussion}

This study evaluated the cost-effectiveness of olaparib and niraparib, 2 of the newly approved PARP inhibitors that have an FDA-approved indication as maintenance therapy in patients with recurrent platinum-sensitive ovarian cancer. Rucaparib data was not included in this study because at the time of analysis it did not have an FDA-approved indication for maintenance therapy. The clinical data were from published pivotal trials, and costs were estimated from standard sources including RED BOOK, Medicare Physician Fee Schedule, and the literature.

The model was constructed from a health care sector perspective and did not include any indirect costs, such as time lost from work due to maintenance therapy. Including indirect costs would have increased total costs and thus would have made ICERs even larger, if the study was conducted from a societal perspective.

A previous study conducted by Smith et al. (2015) reported a similar ICER of $\$ 258,864$ per PFS life-year for olaparib maintenance therapy compared with placebo in patients with a gBRCA mutation based on a phase 2 study and also concluded that olaparib was not cost-effective. ${ }^{18}$ Our study estimated a slightly lower ICER of 226K per PFS life-year. The difference was at least in part because Smith et al. used only olaparib Study 19 (NCT00753545), the result of which was available at that time, to populate the model, while our study used the results from olaparib Study 19 as well as SOLO-2 (NCT01874353). SOLO-2 reported longer median PFS life-years in the gBRCA cohort (21 months) compared with Study 19 (11.2 months). Regardless, both studies suggested that the ICER for olaparib was well above a threshold of \$50K-100K per PFS life-year. Our study also found that at base case, olaparib was dominated by niraparib in patients with a gBRCA mutation, suggesting that niraparib might be a better choice than olaparib from a costeffectiveness point of view.

The costs of olaparib and niraparib are over $\$ 13 \mathrm{~K}$ for a 30-day supply based on WAC price, but they are not alone, with new cancer drugs commonly priced at $\$ 100 \mathrm{~K}$ per year or higher. In a previous study, the average ICER reported for cancer drugs was more than twice the average ICER for noncancer drugs. ${ }^{19}$ As a result, adopting these cancer drugs into practice has significantly increased costs in cancer care for the health care sector. ${ }^{20-22}$ The high costs are often passed on to patients in the form of premiums or copayments. Cancer drugs are often listed in a separated specialty tier under most health plans, with an average $21 \%-22 \%$ coinsurance. ${ }^{23}$ These costs impose a significant financial burden on patients and their families. In addition, as drug costs increase, patients delay or skip cancer treatments. To help, several organizations, including the
American Society of Clinical Oncology, the European Society for Medical Oncology, the Institute for Clinical and Economic Review, and the National Comprehensive Cancer Network, have developed frameworks to systematically assess the value of new drugs against clinical benefits, risks, and costs or affordability. ${ }^{20,24-29}$ As new and innovative health technologies bring hope to cancer patients and their families, there needs to be mechanisms and supports to help pay for the additional costs.

\section{Limitations}

Our model had several limitations. First, the decision tree required a few assumptions. For example, when estimating costs associated with grade 3 or 4 AEs, the costs were not directly from the trials but from literature estimations.

Second, assumptions had to be made about the HSUVs in different treatment groups. The trials we used to inform the decision model did not report EuroQol 5D data, a common utility measure. The HSUVs used in this study were obtained from a study that mapped HSUVs using results of the FACT-Ovarian questionnaire collected in olaparib Study 19 (NCT00753545), a placebo-controlled phase 2 trial of olaparib as a maintenance therapy in recurrent ovarian cancer patients. ${ }^{30}$ The HSUVs for patients on niraparib were not available at the time of this study analysis. Therefore, the base-case analysis did not include HSUVs, which were only included as part of the sensitivity analysis. The HSUVs that were mapped to patients with and without gBRCA mutations were applied to the treatment arms and the placebo, since no study-specific utility measures were reported. Also, no significant difference in health-related quality of life between treatment arms and placebo for these drugs was reported, despite the association of PARP inhibitors with higher rates of AEs, including nausea, fatigue, headache, and significant hematological abnormalities. These parameters represent the best available evidence but are associated with considerable uncertainty. Including them in the sensitivity analysis allowed us to assess the QA-PFS. Varying the HSUVs in the sensitivity analyses indicated that these parameters did not significantly affect overall costeffectiveness.

Finally, because of the relatively new introduction of PARP inhibitors to clinical practice, evidence is still limited with regard to the overall survival of patients who are prescribed these drugs. PFS was used as the outcome measure because it was the primary endpoint in all of the included studies. At the time of this study, overall survival data on PARP inhibitor maintenance therapy was only available for olaparib as a secondary endpoint after more than 5 years follow-up in Study 19 (NCT00753545). ${ }^{31}$ Patients with gBRCA mutations who were receiving olaparib appeared to have longer overall survival compared with placebo, but the result was not statistically significant. For SOLO-2 and NOVO trials, the overall survival data were not available at the time of this study, since the 


\section{Cost-Effectiveness of Niraparib and Olaparib as Maintenance Therapy for Patients with Platinum-Sensitive Recurrent Ovarian Cancer}

extension studies were still ongoing. Therefore, incremental cost-effectiveness for life-years or quality-adjusted life-years was not evaluated.

\section{Conclusions}

Olaparib and niraparib as maintenance therapy may significantly extend PFS in patients with platinum-sensitive recurrent ovarian cancer..$^{10-12}$ However, these drugs are also associated with significant costs from a health care sector perspective. Most of the costs come from drug costs and management of toxicity. This study demonstrated that at base case, niraparib was associated with slightly more PFS benefits than olaparib, but it also cost more than olaparib. When compared with common baseline placebo, both drugs had ICERs of over $\$ 200 \mathrm{~K}$ per PFS life-year. The ICERs were around \$300K per QA-PFS lifeyear when we incorporated HSUVs. In general, use of olaparib or niraparib in patients with $g B R C A$ mutations is more costeffective than in patients without $g B R C A$ mutations. However, the conclusion of the cost-effectiveness analysis is that both drugs are not considered to be cost-effective options.

\section{Authors}

LIXIAN ZHONG, PhD; ANH THU TRAN, BS; and TAYLOR TOMASINO, BS, Irma Lerma Rangel College of Pharmacy, Texas A\&M University, College Station. ELIZABETH NUGENT, MD, and JUDITH A. SMITH, PharmD, BCOP, CPHQ, Department of Obstetrics, Gynecology and Predictive Sciences, The University of Texas Health Science Center at Houston.

AUTHOR CORRESPONDENCE: Lixian Zhong, PhD, Assistant Professor, Irma Lerma Rangel College of Pharmacy, Texas AEM University, 1114 TAMU, College Station, TX 77843-0000. Tel.: 979.436.0193; E-mail: zhong@pharmacy.tamhsc.edu.

\section{DISCLOSURES}

This study was unfunded. The authors have nothing to disclose.

\section{REFERENCES}

1. Centers for Disease Control and Prevention. Ovarian cancer epidemiology in the U.S. 2017. Available at: https://www.cdc.gov/cancer/knowledge/provider-education/ovarian/epidemiology.htm. Accessed September 28, 2018.

2. National Cancer Institute, Surveillance, Epidemiology, and End Results Program. Cancer stat facts: ovarian cancer. 2017. Available at: https://seer. cancer.gov/statfacts/html/ovary.html. Accessed September 28, 2018.

3. Siegel RL, Miller KD, Jemal A. Cancer statistics, 2018. CA Cancer J Clin. 2018;68(1):7-30.

4. Prat J; FIGO Committee on Gynecologic Oncology. Staging classification for cancer of the ovary, fallopian tube, and peritoneum. Int J Gynaecol Obstet. 2014;124(1):1-5.

5. McMeekin DS, Tillmanns T, Chaudry T, et al. Timing isn't everything: an analysis of when to start salvage chemotherapy in ovarian cancer. Gynecol Oncol. 2004:95(1):157-64.
6. Herzog TJ, Armstrong DK. First-line chemotherapy for advanced (stage III or IV) epithelial ovarian, fallopian tubal, and peritoneal cancer. Vora SR, ed. UpToDate. August 27, 2017. Available at: https://www.uptodate.com/ contents/first-line-chemotherapy-for-advanced-stage-iii-or-iv-epithelial-ovarian-fallopian-tubal-and-peritoneal-cancer?source=see_link\#H760206841. Accessed September 28, 2018.

7. Herzog TJ, Holloway RW, Stuart GC. Workshop: options for therapy in ovarian cancer. Gynecol Oncol. 2003;90(3 Pt 2):S45-50.

8. Aghajanian C, Blank SV, Goff BA, et al. OCEANS: a randomized, double-blind, placebo-controlled phase III trial of chemotherapy with or without bevacizumab in patients with platinum-sensitive recurrent epithelial ovarian, primary peritoneal, or fallopian tube cancer. J Clin Oncol. 2012;30(17):2039-45.

9. Coleman RL, Brady MF, Herzog TJ, et al. Bevacizumab and paclitaxel-carboplatin chemotherapy and secondary cytoreduction in recurrent, platinumsensitive ovarian cancer (NRG Oncology/Gynecologic Oncology Group study GOG-0213): a multicentre, open-label, randomised, phase 3 trial. Lancet Oncol. 2017;18(6):779-91

10. Ledermann J, Harter P, Gourley C, et al. Olaparib maintenance therapy in platinum-sensitive relapsed ovarian cancer. N Engl J Med. 2012;366(15):1382-92.

11. Pujade-Lauraine E, Ledermann JA, Selle F, et al. Olaparib tablets as maintenance therapy in patients with platinum-sensitive, relapsed ovarian cancer and a BRCAl/2 mutation (SOLO2/ENGOT-Ov21): a doubleblind, randomised, placebo-controlled, phase 3 trial. Lancet Oncol. 2017;18(9):1274-84.

12. Mirza MR, Monk BJ, Herrstedt J, et al. Niraparib maintenance therapy in platinum-sensitive, recurrent ovarian cancer. N Engl J Med. 2016;375(22):2154-64.

13. Truven Health Analytics, IBM Watson. Micromedex RED BOOK. Database. 2017. Available at: http://truvenhealth.com/Products/Micromedex/ProductSuites/Clinical-Knowledge/RED-BOOK. Accessed September 28, 2018.

14. Mansley EC, Carroll NV, Chen KS, et al. Good research practices for measuring drug costs in cost-effectiveness analyses: a managed care perspective: the ISPOR Drug Cost Task Force report-Part III. Value Health. 2010;13(1):14-17.

15. Bureau of Labor Statistics. Consumer Price Index for medical care. 2017. Available at: https://www.bls.gov/cpi/data.htm. Accessed October 16, 2018.

16. U.S. Department of Health and Human Services. Common terminology criteria for adverse events (CTCAE). Version 5.0. November 27, 2017. Available at: https://ctep.cancer.gov/protocoldevelopment/electronic_applications/docs/ CTCAE_v5_Quick_Reference_5x7.pdf. Accessed September 28, 2018.

17. Neumann PJ, Cohen JT, Weinstein MC. Updating cost-effectivenessthe curious resilience of the \$50,000-per-QALY threshold. N Engl J Med. 2014;371(9):796-97.

18. Smith HJ, Walters Haygood CL, Arend RC, Leath CA 3rd, Straughn JM Jr. PARP inhibitor maintenance therapy for patients with platinum-sensitive recurrent ovarian cancer: a cost-effectiveness analysis. Gynecol Oncol. 2015;139(1):59-62.

19. Bae YH, Mullins CD. Do value thresholds for oncology drugs differ from nononcology drugs? J Manag Care Spec Pharm. 2014;20(11):1086-92. Available at: https://www.jmcp.org/doi/10.18553/jmcp.2014.20.11.1086.

20. Chandra A, Shafrin J, Dhawan R. Utility of cancer value frameworks for patients, payers, and physicians. JAMA. 2016;315(19):2069-70.

21. Ramsey SD, Lyman GH, Bangs R. Addressing skyrocketing cancer drug prices comes with tradeoffs: pick your poison. JAMA Oncology. 2016;2(4):425-26.

22. Salas-Vega S, Mossialos E. Cancer drugs provide positive value in nine countries, but the United States lags in health gains per dollar spent. Health Aff (Millwood). 2016;35(5):813-23. 


\section{Cost-Effectiveness of Niraparib and Olaparib as Maintenance Therapy for Patients with Platinum-Sensitive Recurrent Ovarian Cancer}

23. EMD Serono. EMD Serono specialty digest: managed care strategies for specialty pharmaceuticals. 14th ed. 2018. Available at: https://specialtydigestemdserono.com/. Accessed October 16, 2018

24. Schnipper LE, Davidson NE, Wollins DS, et al. American Society of Clinical Oncology statement: a conceptual framework to assess the value of cancer treatment options. J Clin Oncol. 2015;33(23):2563-77.

25. Schnipper LE, Davidson NE, Wollins DS, et al. Updating the American Society of Clinical Oncology value framework: revisions and reflections in response to comments received. J Clin Oncol. 2016;34(24):2925-34.

26. National Comprehensive Cancer Network. NCCN Clinical Practice Guidelines in Oncology with Evidence Blocks. Available at: https://www. nccn.org/evidenceblocks/. Accessed September 28, 2018.

27. Cherny NI, Sullivan R, Dafni U, et al. A standardised, generic, validated approach to stratify the magnitude of clinical benefit that can be anticipated from anti-cancer therapies: the European Society for Medical Oncology Magnitude of Clinical Benefit Scale (ESMO-MCBS). Ann Oncol. 2015;26(8):1547-73.

28. Ollendorf D, Pearson SD. ICER evidence rating matrix: a user's guide. 2016. Available at: http://www.amcp.org/ICERUserGuide.pdf. Accessed September 28, 2018.

29. Bentley TGK, Cohen JT, Elkin EB, et al. Measuring the value of new drugs: validity and reliability of 4 value assessment frameworks in the oncology setting. J Manag Care Spec Pharm. 2017;23(6-a Suppl):S34-48. Available at: https://www.jmcp.org/doi/10.18553/jmcp.2017.23.6-a.s34.

30. Ledermann JA, Harter P, Gourley C, et al. Quality of life during olaparib maintenance therapy in platinum-sensitive relapsed serous ovarian cancer. Br J Cancer. 2016;115(11):1313-20.
31. Ledermann JA, Harter P, Gourley C, et al. Overall survival in patients with platinum-sensitive recurrent serous ovarian cancer receiving olaparib maintenance monotherapy: an updated analysis from a randomised, placebocontrolled, double-blind, phase 2 trial. Lancet Oncol. 2016;17(11):1579-89.

32. Centers for Medicare \& Medicaid Services. Medicare physician fee schedule look-up tool. August 15, 2017. Available at: https://www.cms.gov/ apps/physician-fee-schedule/overview.aspx. Accessed September 28, 2018.

33. Calhoun EA, Chang CH, Welshman EE, Fishman DA, Lurain JR, Bennett CL. Evaluating the total costs of chemotherapy-induced toxicity: results from a pilot study with ovarian cancer patients. Oncologist. 2001;6(5):441-45.

34. Suidan RS, He W, Sun CC, et al. Treatment patterns, outcomes, and costs for bowel obstruction in ovarian cancer. Int J Gynecol Cancer. 2017;27(7):1350-59.

35. Swisher EM, Lin KK, Oza AM, et al. Rucaparib in relapsed, platinumsensitive high-grade ovarian carcinoma (ARIEL2 Part 1): an international, multicentre, open-label, phase 2 trial. Lancet Oncol. 2017;18(1):75-87.

36. Cancer Genome Atlas Research Network. Integrated genomic analyses of ovarian carcinoma. Nature. 2011;474(7353):609-15.

37. Pennington KP, Walsh T, Harrell MI, et al. Germline and somatic mutations in homologous recombination genes predict platinum response and survival in ovarian, fallopian tube, and peritoneal carcinomas. Clin Cancer Res. 2014;20(3):764-75

38. Ledermann J, Harter P, Gourley C, et al. Olaparib maintenance therapy in patients with platinum-sensitive relapsed serous ovarian cancer: a preplanned retrospective analysis of outcomes by BRCA status in a randomised phase 2 trial. Lancet Oncol. 2014;15(8):852-61. 